\title{
Participação do Pai no Cuidar da Criança com Doença Crônica
}

\author{
Father's Participation in Caring for Children with Chronic Disease
}

\section{RESUMO}

Objetivo: Investigar o nível de participação do pai no cuidado da criança com doença crônica de origem neurológica e/ou genética no tocante às atividades cotidianas e no seu envolvimento em atividades terapêuticas. Metodologia: Pesquisa de campo, de natureza exploratório-descritiva com abordagem quantitativa. O estudo envolveu 30 pais de crianças com paralisia cerebral ou Síndrome de Down, com idade superior a 18 anos. Foi utilizada a análise descritiva e exploratória de dados, testes de associação de qui-quadrado e o teste exato de Fisher, para medir as associações entre as variáveis. Resultados: Verificou-se que $56,67 \%$ dos pais ficavam em casa parte do dia e, desse tempo, $46,34 \%$ destinavam para ficar com a criança. $79,07 \%$ dos pais utilizavam esse tempo para ajudar, assistir TV, passear ou brincar. $66,04 \%$ tinham como responsabilidade, no mínimo, duas tarefas com a criança. $43,33 \%$ não conseguiam conciliar o emprego com o tratamento do filho. Todos se enxergavam como um bom pai, tendo como justificativa a provisão e a amorosidade $(54,76 \%)$. Conclusão: A participação do pai foi notória, ainda que tímida, estando na dependência de sua atividade laboral para não deixar faltar o provimento da família, sendo esta última condição o esperado na visão mais tradicional da paternidade. No que se refere à participação no processo de reabilitação do filho, o pai ainda tem uma participação mínima e/ou nenhuma, no entendimento de que o tempo dedicado ao trabalho ainda é o principal motivo que dificulta seu envolvimento de forma efetiva.

\section{DESCRITORES}

Doença Crônica. Infância. Síndrome de Down. Paralisia Cerebral. Paternidade.

\begin{abstract}
Objective: To investigate the level of father participation in the care of the child with chronic disease of neurological and/or genetic origin regarding daily activities and their involvement in therapeutic activities. Methodology: Field research, exploratory-descriptive nature with a quantitative approach. The study involved 30 parents of children with Cerebral Palsy or Down Syndrome, aged over 18 years. Descriptive and exploratory data analysis and chi-square association tests were used and, to measure associations between variables, whenever necessary, Fisher's exact test was used. Results: $56.67 \%$ of fathers stay at home part of the day, and $46.34 \%$ of that time they spend with the child. $79.07 \%$ of fathers use this time to help, watch TV, go for a walk or play. $66.04 \%$ are responsible for at least two tasks with the child. $43.33 \%$ are unable to balance employment with their child's treatment. All of them see themselves as a good father, justifying it with giving provision and love (54.76\%). Conclusion: The father's participation is notorious, even if in a shy way, being on the premises of his work activity so as not to miss the family's provision. This last condition being expected in the more traditional view of fatherhood. Regarding the participation in the son's rehabilitation process, the father still has minimal and/or no participation, in the understanding that the time dedicated to work is still the main reason that hinders his involvement effectively.
\end{abstract}

\section{DESCRIPTORS}

Chronic Disease. Childhood. Down Syndrome. Cerebral Palsy. Paternity.

\footnotetext{
1. Fisioterapeuta membro externo do NESDI/UFPB, João Pessoa-PB, Brasil.

2. Docente do Departamento de Fisioterapia da UFPB, João Pessoa-PB, Brasil.

3. Docente do Departamento de Fisioterapia da UFPB, Professor do Programa de Pós-Graduação em Fisioterapia da UFPB, João Pessoa-PB, Brasil.
} 
A doença crônica na infância pode ser definida a partir da necessidade de suporte para o desenvolvimento motor e da comunicação contribuindo para a interação com o meio, com as pessoas e a compreensão de expressões afetivas, desejos e vontades da criança ${ }^{1}$. A paralisia cerebral (PC), assim como a síndrome de Down (SD) são condições neurológicas que têm em comum um processo crônico e que traz consigo limitações que impactam no cotidiano das crianças e de seus familiares ${ }^{2}$.

A PC, considerada a incapacidade física mais comum na infância, é definida como um grupo de desordens do movimento e da postura, que causam limitação em atividades cotidianas e que são atribuídas a distúrbios não progressivos ocorridos durante o desenvolvimento do cérebro imaturo ${ }^{3}$. Enquanto, a síndrome de Down é o resultado de um acidente genético causado pela trissomia do cromossomo 21 , que resulta em características físicas e mentais específicas, sendo a causa genética mais comum de deficiência mental ${ }^{4}$. No caso dessas duas situações, o comprometimento se dá na infância e os cuidados com a saúde e o desenvolvimento neuropsicomotor são necessários por toda vida, o que leva a uma demanda por parte dos pais, em busca de serviços de saúde e uma construção de rede que abranja a família, o hospital, a escola e o sistema de garantia de direitos $^{5}$

O pai e a mãe precisam estar presentes no cotidiano da criança com algum comprometimento neurológico, não apenas, para garantir a assistência continuada a seus filhos, mas também são primordiais para torná-los a cada dia, mais ativos e participativos na vida escolar, no lazer e no ambiente familiar.
Na perspectiva da Teoria Bioecológica de Bronfenbrenner, a relação pessoa/contexto, no caso a criança com doença crônica e seus pais/ambiente familiar, é fundamental para o desenvolvimento humano e é considerado um processo interativo e bidirecional ${ }^{6}$. Nesta teoria, o nível chamado de microssistema, representado aqui pelo núcleo familiar, refere-se ao lócus onde os pais estão inseridos e no qual a criança passa a maior parte do seu tempo e participa ativamente. Por isso deve ser considerado um ambiente importante na promoção do desenvolvimento ${ }^{6,7}$.

O vínculo entre a mãe e o filho é o relacionamento mais fortemente entrelaçado pelo cuidar do outro, é a mãe que, mais frequentemente, passa a ser a principal cuidadora da criança com $\mathrm{PC}$ e SD ${ }^{8}$. No entanto, como esse cuidar demanda tempo e contínua dedicação, a mãe precisa dá colaboração de outros familiares. O pai, em grande parte dos casos, mantém-se ausente devido à reponsabilidades e exigências do trabalho, principalmente, quando desempenha o papel de provedor da família 9 .

Os estudos sobre o desenvolvimento de crianças com doença crônica ou deficiência ficam centrados na perspectiva da mãe e nas informações dadas por esta ${ }^{10}$. Ainda se sabe pouco sobre a percepção paterna acerca de crianças que têm o desenvolvimento comprometido, menos ainda sobre sua relação com o cuidado delas ${ }^{8,9,11}$ A ausência dos pais ou a tendência em deixá-lo em segundo plano, desconsidera o importante papel desempenhado por eles dentro do grupo familiar. Assim, existe a necessidade crescente de se compreender qual a participação do pai nas relações e tarefas familiares, especialmente relacionadas ao 
desenvolvimento físico/emocional e a reabilitação de filhos com necessidades especiais?

Diante do exposto, o estudo objetiva compreender como ocorre a participação do pai no cuidado da criança com doença crônica de origem neurológica e/ou genética; e para tal, traçou-se um perfil sociodemográfico da amostra e investigou-se a atuação paterna junto às atividades cotidianas do filho e o seu envolvimento em atividades terapêuticas.

\section{METODOLOGIA}

Trata-se de um estudo transversal, de natureza exploratório-descritiva e com abordagem quantitativa. Participaram da pesquisa indivíduos do sexo masculino, com idade maior que 18 anos, cujos filhos estavam em atendimento em serviços públicos especializados de fisioterapia, na Cidade de João Pessoa, na Paraíba, nos casos em que os diagnósticos tinham sido estabelecidos como síndrome de Down ou paralisia cerebral. Portanto, trata-se de uma amostra por conveniência que totalizou 30 pais.

Utilizou-se um instrumento adaptado de Silva ${ }^{12}$, a partir de uma entrevista semi-estruturada, contendo informações sobre o perfil socioeconômico da família, o tempo de interação pai-filho, as atividades paternas no cuidar da criança e as percepções sobre a paternidade. $O$ contato com a maioria dos pais foi obtido por meio das mães. Essa estratégia se deu pelo fato de ser a mãe quem, normalmente, levava a criança ao tratamento.

Após assinatura do termo de consentimento livre e esclarecido pelos participantes que aceitaram participar da pesquisa, em atenção a Resolução 466/2012, foram reali- zadas as entrevistas. Como a pesquisa tem como risco expor informações pessoais dos pais, tornou-se como medida a preservação da identidade e nomes dos participantes. Posteriormente os dados coletados foram tabulados e submetidos à análise estatística descritiva e inferencial com o software Package for the Social Sciences (SPSS $®$ ), versão 20.0 .

Para a caracterização dos participantes da pesquisa foram utilizadas as medidas de frequência para variáveis categóricas, a média e o desvio padrão para variáveis numéricas. O teste do qui-quadrado foi utilizado para verificar a associação entre as variáveis categóricas, fixando um nível de significância de $5 \%$ e sempre que foi necessário, o teste exato de Fisher mediu a associação entre as variáveis para aquelas situações em que o teste de associação do qui-quadrado não pode ser aplicado.

\section{RESULTADOS}

Caracterização sociodemográfica dos participantes da pesquisa

Dos 30 entrevistados, 12 eram pais de crianças com paralisia cerebral, com média de idade de 41,33 $\pm 9,33$ anos; e 18 eram genitores de crianças com síndrome de Down, com idade média de 38,28 $\pm 7,88$ anos. Algumas questões como a situação atual de emprego, o estado civil, a condição de moradia e a renda familiar foram abordados para contextualizar a situação familiar dos participantes e estão dispostas na Tabela 1.

Em relação aos testes de associação do perfil demográfico dos pais com o tipo de doença da criança revelaram, com exceção 
Tabela 1. Caraterísticas sociodemográficas da amostra de acordo com o diagnóstico da criança.

\begin{tabular}{|c|c|c|c|c|c|c|c|}
\hline \multirow{4}{*}{$\begin{array}{l}\text { Perfil } \\
\text { Média/desvio padrão } \\
\text { Idade da criança (filhos) } \\
\text { Frequência /porcentagem }\end{array}$} & \multicolumn{2}{|c|}{$\begin{array}{c}\text { Paralisia cerebral } \\
\quad(n=12)\end{array}$} & \multicolumn{2}{|c|}{$\begin{array}{c}\text { Síndrome Down } \\
\quad(n=18)\end{array}$} & \multicolumn{2}{|c|}{ Total $(n=30)$} & $\begin{array}{l}\text { Significância } \\
\text { (1)(Valor-p) }\end{array}$ \\
\hline & \multicolumn{2}{|c|}{$\mathrm{M} \pm \mathrm{DP}$} & \multicolumn{2}{|c|}{$\mathrm{M} \pm \mathrm{DP}$} & \multicolumn{2}{|c|}{$\mathrm{M} \pm \mathrm{DP}$} & \\
\hline & \multicolumn{2}{|c|}{$9,67 \pm 4,31$} & \multicolumn{2}{|c|}{$5,78 \pm 3,54$} & \multicolumn{2}{|c|}{$7,33 \pm 4,26$} & $p^{(1)}=0,0172$ \\
\hline & $\mathrm{n}$ & $\%$ & $\mathrm{n}$ & $\%$ & $\mathrm{n}$ & $\%$ & \\
\hline \multicolumn{8}{|l|}{ Sexo (criança) } \\
\hline Feminino & 03 & 25,00 & 11 & 61,11 & 14 & 46,67 & \multirow{2}{*}{$p^{(2)}=0,0521$} \\
\hline Masculino & 09 & 75,00 & 07 & 38,89 & 16 & 53,33 & \\
\hline \multicolumn{8}{|l|}{ Número de filhos } \\
\hline 01 filho & 03 & 25,00 & 07 & 38,89 & 10 & 33,33 & \multirow{2}{*}{$p^{(2)}=0,4292$} \\
\hline 02 ou + filhos & 09 & 75,00 & 11 & 61,11 & 20 & 66,67 & \\
\hline \multicolumn{8}{|l|}{ Empregado } \\
\hline Sim & 08 & 66,67 & 11 & 61,11 & 19 & 63,33 & \multirow{2}{*}{$\mathrm{p}^{(2)}=0,7571$} \\
\hline Não & 04 & 33,33 & 07 & 38,89 & 11 & 36,67 & \\
\hline \multicolumn{8}{|l|}{ Situação financeira } \\
\hline $\begin{array}{l}\text { Trabalha e é o único } \\
\text { provedor da família }\end{array}$ & 08 & 66,67 & 08 & 44,44 & 16 & 53,33 & \multirow{3}{*}{$p^{(3)}=0,6491$} \\
\hline $\begin{array}{l}\text { Sustento da família } \\
\text { proveniente do casal }\end{array}$ & 03 & 25,00 & 08 & 44,44 & 11 & 36,67 & \\
\hline Outro & 01 & 8,33 & 02 & 11,11 & 3 & 10,00 & \\
\hline \multicolumn{8}{|l|}{ Tipo de moradia } \\
\hline Próprio & 8 & 66,67 & 10 & 55,56 & 18 & 60,00 & \multirow{3}{*}{$p^{(3)}=0,8609$} \\
\hline Alugado & 1 & 8,33 & 2 & 11,11 & 3 & 10,00 & \\
\hline Cedido/outro & 3 & 25,00 & 6 & 33,33 & 9 & 30,00 & \\
\hline
\end{tabular}

da idade da criança (que apresentou um valor-p<0,05), que nenhuma característica do perfil demográfico dos pais estava associada com o tipo de condição crônica da criança.

O uso do tempo e dedicação dos pais aos cuidados com a criança

Os pais participantes desse estudo, em se tratando de sua permanência em casa durante o dia, a maioria respondeu que ficava em casa uma parte do dia e que ajudava com as crianças, principalmente, em brincadeiras para distrair as mesmas. Em relação ao tempo dedicado para a reabilitação das crianças com SD, (61,11\%) dos pais afirmaram não ter tempo em participar desse processo. Enquanto, os pais de crianças com PC $(58,33 \%)$ dedicavam tempo entre dois a cinco dias da semana para a reabilitação de seus filhos, os dados aparecem na Tabela 2 de forma mais detalhada.

Com relação aos testes de associação, nenhuma dessas características se mostrou associada significativamente com a situação crônica da criança, uma vez que todos os valores-p dos testes empregados foram maiores que 0,05 .

A rotina dessas crianças, desde o nas- 
cimento, passa por uma série de atendimentos a diversos profissionais e a família empreende todo o esforço possível para acompanhar o ritmo exigido neste processo. No entanto, os pais que estavam empregados, sendo mais de $50 \%$ da amostra, declararam que era muito difícil conciliar o trabalho e as demandas existentes para a reabilitação de seus filhos ou destacaram não conseguir colaborar com o tratamento por causa do trabalho.

Responsabilidades, dificuldades e vivências dos pais com a criança

A entrevista com os participantes revelou que $64 \%$ dos pais de crianças com PC têm como responsabilidades para com a criança, dar a alimentação e pôr a criança para dormir, 86,67\% também afirmaram ter dificuldades financeiras e falta de tempo de manter o tratamento dos filhos; metade deles afirma que tem dificuldade de cuidar das crianças, principalmente porque não a entende ou simplesmente não tem paciência (50\%).
Quando foi perguntado aos participantes sobre a opinião da esposa sobre sua participação como pai na rotina do filho, $72,73 \%$ responderam que poderiam participar da rotina das crianças de outra forma, em especial, sendo mais presente $(18,18 \%)$ ou interagindo mais com a criança $(45,45 \%)$. No outro grupo de pais, àqueles de crianças com SD, $67,86 \%$ deles entendem que as suas responsabilidades para com a criança são, pôr a criança para dormir ou dar banho; $80,95 \%$ dos pais também afirmaram ter dificuldades financeiras e a de falta de tempo de manter o tratamento dos filhos; $33,33 \%$ dos pais informaram que têm dificuldades de cuidar das crianças, sendo a falta de paciência (60\%) e de tempo (40\%) os dois únicos motivos relatados. Adicionalmente, $66,67 \%$ das mães, segundo os pais, entendem que eles poderiam participar da rotina das crianças sendo mais presente $(31,58 \%)$ ou interagindo mais com a criança $(31,58 \%)$.

Tabela 2. Tempo do pai dedicado ao cuidado e atividades desenvolvidas com a criança.

\begin{tabular}{|c|c|c|c|c|c|c|c|}
\hline \multirow{2}{*}{$\begin{array}{l}\text { Uso do tempo e atividades } \\
\text { desenvolvidas }\end{array}$} & \multicolumn{2}{|c|}{$\begin{array}{l}\text { Paralisia cerebral } \\
(n=12)\end{array}$} & \multicolumn{2}{|c|}{$\begin{array}{l}\text { Síndrome Down } \\
(n=18)\end{array}$} & \multicolumn{2}{|c|}{ Total $(n=30)$} & \multirow{2}{*}{ Valor-p } \\
\hline & $n$ & $\%$ & $n$ & $\%$ & $\mathrm{n}$ & $\%$ & \\
\hline \multicolumn{8}{|l|}{ Tempo que fica em casa } \\
\hline A cada 2 dias & 0 & 0,00 & 1 & 5,56 & 1 & 3,33 & \multirow{4}{*}{$p^{(1)}=0,2496$} \\
\hline O dia todo & 4 & 33,33 & 2 & 11,11 & 6 & 20,00 & \\
\hline Metade do dia & 1 & 8,33 & 5 & 27,78 & 6 & 20,00 & \\
\hline Parte do dia & 7 & 58,33 & 10 & 55,56 & 17 & 56,67 & \\
\hline \multicolumn{8}{|l|}{ Uso do tempo com a criança } \\
\hline Ajuda/brinca com a criança & 9 & 52,94 & 15 & 57,69 & 24 & 55,81 & \multirow{4}{*}{$p^{(2)}=0,9169$} \\
\hline Assiste TV/passeia & 5 & 29,41 & 5 & 19,23 & 10 & 23,26 & \\
\hline Coloca a criança para dormir & 1 & 5,88 & 2 & 7,69 & 3 & 6,98 & \\
\hline Dar atenção & 2 & 11,76 & 4 & 15,38 & 6 & 13,95 & \\
\hline
\end{tabular}

(1) Teste de associação de qui-quadrado, (2) Teste exato de Fisher. 
Percepção e autoavaliação da paternidade

Ao serem questionados sobre o papel de pai, $80 \%$ dos pais afirmaram que a paternidade mudou a forma de pensar e de agir. Em relação à autoavaliação do papel de ser pai, $62,5 \%$ dos pais das crianças com PC disseram que são bons pais porque são provedores do lar, pacientes e responsáveis. Entretanto, entre os pais de crianças com SD, a amorosidade e a busca por fazer o melhor sempre são as justificativas da boa paternidade para esse grupo.

\section{DISCUSSÃO}

Antes relegados a segundo plano no campo de estudos sobre a família e o desenvolvimento infantil, os pais vêm ganhando, nas últimas décadas, significativa atenção, principalmente sob o olhar de estudiosos do desenvolvimento humano ${ }^{9,10}$ Para a teoria bioecológica, a força motriz do desenvolvimento infantil são os processos que ocorrem mais proximais, como no ambiente familiar, onde a mãe e o pai exercem papéis fundamentais, por causa do apego emocional mútuo e forte com as crianças ${ }^{13}$.

Neste contexto, os cuidados e desafios que envolvem uma criança com doença neurológica são enormes, principalmente porque exige de toda a família uma reestruturação das ações, dos hábitos e do tempo para atender as necessidades das crianças. Existe neste caso, não apenas a questão do cuidar da saúde do filho, mas também a necessidade de manter relacionamentos sustentadores, contínuos como processo de interações afetuosas, seguras, empáticas e que demandam carinho e paciência redobrada entre os pais e a criança ${ }^{5,2}$.
No presente estudo, observou-se que os pais, em sua maioria, trabalhavam e buscavam participar de alguma forma do desenvolvimento, do cuidar e do tratamento de seus filhos. O grande desafio nessa participação é praticamente diário, porque as demandas das crianças com PC e SD são intensas e variadas, necessitando de ajustes entre os horários de trabalho e a colaboração com a família.

A influência do trabalho dos pais sobre a relação com os filhos, foi um ponto bem destacado no atual estudo e, de certa maneira, o tipo de emprego e a carga horária de trabalho foram razões para a ocorrência de baixos níveis de envolvimento ou participação paterna. Cuidar de crianças, como nos casos de PC e $S D$, requer atenção constante, não existindo tempo disponível suficiente para associar o trabalho fora de casa e o cuidado delas ${ }^{14}$. Mesmo com uma amostra heterogênea em relação ao trabalho, visto que cerca de $30 \%$ estavam desempregados, os pais afirmaram participar de atividades como a alimentação, pôr para dormir, dar banho, levar/buscar no médico e na escola.

Alguns outros aspectos foram destacados pelos pais participantes, relativo à dificuldades que sentem no cuidar de seus filhos, que foram: não entende a criança, não tem paciência, a falta de tempo e muito trabalho. A chegada à família de uma criança com SD ou com PC, esta se torna o centro de sentimentos, dúvidas, incertezas e, principalmente, de medo do desconhecimento do que essas condições neurológicas possam levar seus filhos a sofrerem ${ }^{15}$.

A partir dessa realidade, a teoria de Bronfenbrenner aponta para a necessidade 
de existir uma interação entre os ambientes que a criança se desenvolve e, para isto, os pais não podem se privar do apoio de sua rede social, a qual poderá colaborar no cuidado à criança com necessidades especiais ${ }^{6,13}$. Embora parte da amostra $(58,33 \%)$ referente aos pais de crianças com PC, relatou participar semanalmente da reabilitação de seus filhos, é preciso ressaltar, que acompanhar os filhos às consultas e aos tratamentos necessários, nem sempre significa adentrar nos consultórios: muitos pais simplesmente transportam-nos. Algumas vezes, o pai acompanha a criança até a sala de espera e não participa dos atendimentos; porém, quando entra no consultório, muitas vezes, não lhe são dirigidas perguntas, uma vez que o hábito é dirigir-se basicamente à mãe, principalmente, quando o filho ainda é muito pequeno ${ }^{16}$.

Ainda sobre outros aspectos abordados no questionário sobre a responsabilidade da alimentação e pôr a criança para dormir, os resultados convergem com os achados de outros estudos ${ }^{17}$, que apontam para o fato de que os pais vêm se incluindo timidamente nos cuidados da criança. Uma vez que contribuem no cuidado, mas ainda de forma simples, tomando para si responsabilidades básicas, que não exigem tanto tempo ou esforço. Contudo, no presente estudo, na visão dos pais sobre a opinião das mães, eles poderiam participar da rotina das crianças de outra forma, em especial, sendo mais presentes ou interagindo mais com a criança. Portanto, é preciso ter cautela ao atribuir ao pai a ausência ou o afastamento do filho e da mãe, pois pai e mãe encontram, nesta relação, um terreno fértil para que isto ocorra, uma vez que a mãe toma para si a tarefa de cuidar, deixando pouco espaço para que o pai divida com ela esta responsabilidade na relação, ação esta reforçada pelos profissionais da área, que, ao se dirigirem, o fazem prioritariamente à mãe ${ }^{16}$.

A questão da influência e autoavaliação da paternidade retratam dois questionamentos simples feitos aos pais: como a paternidade influenciou a vida dele e como ele se avalia como pai. Essas duas questões são primordiais, pois reconstroem o papel primitivo do pai na composição e no bem-estar da família, no que se refere ao suporte à criança com deficiência. De acordo com o que os dados mostram, a paternidade mudou a forma de pensar e a forma de agir dos pais. $\mathrm{Na}$ autoavaliação do papel desempenhado, todos se enxergam como um bom pai, tendo como justificativa a provisão e a amorosidade. Tal condição sugere que o pai, à sua maneira, se julga presente na relação e no cuidado com o filho, mesmo frente ao pouco tempo que fica com a criança.

\section{CONCLUSÃO}

Os resultados do estudo mostraram que a participação do pai foi notória, ainda que de forma tímida, ele tem dado a sua contribuição, buscando participar da rotina da criança em casa sem deixar de lado o provimento das necessidades da família, sendo esta última condição, o esperado na visão mais tradicional da paternidade.

No que se refere à contribuição/participação com o processo de reabilitação do filho, o pai ainda tem uma participação mínima e/ou nenhuma, no entendimento de que o tempo dedicado ao trabalho ainda é o principal motivo que dificulta seu envolvimento de forma efetiva.

Considera-se, neste estudo, o quanto 
se faz necessária a participação paterna no processo de reabilitação das crianças acometidas por agravos crônicos no entendimento de que, a participação da mulher ainda é relevante nesse processo, tendo a mesma mais uma atribuição, se pensarmos no processo histórico em que se elenca inúmeras responsabilidades à mulher.

Conclui-se entendendo a necessidade de mais pesquisas que priorizem o pai como objeto de estudo e que deem voz a ele, pois

\section{REFERÊNCIAS}

1. Moreira MCN, Gomes R, Sá MRC de. Doenças Crônicas em crianças e adolescentes: uma revisão bibliográfica. Ciência e Saúde Coletiva. 2014; 19(7): 2018-2094.

2. Santos KH , Marques D, Souza, AC. Crianças e adolescentes com paralisia cerebral: análise sobre longitudinalidade do cuidado. Texto Contexto Enferm. 2017; 26(2):1-9.

3. Cavalcante VMV, Martins MC, Oriá MOB, Ximenes LB, Frota MA, Carvalho ZMF. Perfil epidemiológico das crianças com paralisia cerebral em atendimento ambulatorial. Rev enferm UERJ. 2017; 25:1-7.

4. Hannum JSS, Miranda FJ, Salvador IF, Cruz AP. Impacto do diagnóstico nas famílias de pessoas com síndrome de down: revisão da literatura. Pensando Famílias. 2018; 22(2):121-136.

5. Cunha KC, Pontes Far, Silva SSC. Pais de crianças com paralisia cerebral pouco estressados. Rev. Bras. Ed. Esp. 2017; 23(1):111-126.

6. Lemos RA, Verissímo MLÓR, Desenvolvimento de crianças nascidas prematuras: a compreensão dos cuidadores à luz da Teoria Bioecológica. Rev Esc Enferm USP. 2015; 49(6):899-907.

7. Batista JMS, Trigueiro TH, Lenardt MH, Mazza VA, Labronici LM. O modelo bioecológico: desvendando contribuições para a práxis da enfermagem diante da violência doméstica. Esc Anna Nery (impr.). 2013; 17(1):173-178.

8. Barros ALO, Barros AO, Barros GLM, Santos MTBR. Sobrecarga dos cuidadores de crianças e adolescentes com Síndrome de Down. Ciência e Saúde Coletiva. 2017; 22(11):625-634.

9. Silva DCMS, Reichert APS, Nóbrega MN, Dantas MAS, Gomes GLL, Macedo J Q de, Pimenta EAG, Torquato IMB, Collet N. Difficulties in a Father's Day-to-Day Care of a Child with A Chronic Illness. International Arch of Medicine. 2016; 9(143):1-9.

10. Ramos RM, Nóbrega VM, Fernandes LTB, Machado NA, Collet $\mathrm{N}$. Cuidado paterno à criança e ao adolescente com doença crônica: percepção materna. Rev Gaúcha Enferm. 2017; 38(3):1-8. muito do que tem circulado na literatura a respeito da representação social do pai, configura-se como mero mito ou sofre o viés daqueles que falam em nome do pai. Desta feita, estudos nesta área podem fornecer subsídios para implementar programas de intervenção interdisciplinar às famílias de crianças com doença crônica, visando melhorar a interação entre os seus membros, promovendo uma rotina familiar de melhor qualidade e de aceitação desta criança.

11. Canho, PGM, Neme, CMB, Yamada, MO. A vivência do pai no processo de reabilitação da criança com deficiência auditiva. Estudos de Psicologia. 2006; 23(3): 261-269.

12. Silva, NCB. Intervenção domiciliar e envolvimento paterno: Efeitos em famílias de crianças com síndrome de Down. [Tese de Doutorado]. São Paulo: Universidade Federal de São Carlos, 2012

13. Neta Santiago IS, Medeiros MS, Gonçalves MJF. Vigilância da saúde orientada às condições de vida da população: uma revisão integrativa da literatura. 2018; 42(116):307-317.

14. Marx C, Rodrigues EM, Rodrigues MM, Vilanova LCP. Depressão, ansiedade e sonolência diurna em cuidadores primários de crianças com paralisia cerebral. Rev Paul Pediatr. 2011; 29(4):483-488.

15. Simões CC, Silva L, Santos MR, Misko MD, Bousso RS. A experiência dos pais no cuidado dos filhos com paralisia cerebral. Rev. Eletr. Enf. 2013; 15(1):138-145.

16. Chacon MCM. Aspectos relacionais, familiares e sociais da relação pai-filho com deficiência física. Relação pai-filho e deficiência. Rev. Bras. Ed. Esp. 2011; 17(3): 441-458.

17. Jager ME, Bottoli C. Paternidade: vivência do primeiro filho e mudanças familiares. Psicologia: Teoria e Prática. 2011;13(1):141-153.

18. BRASIL. Ministério da Saúde. Diretrizes de atenção à pessoa com Síndrome de Down. Brasília: Ministério da Saúde/Programa Nacional de Atenção a Pessoa Portadora de Deficiência; 2013.

\section{CORRESPONDÊNCIA}

Sandra Maria Cordeiro Rocha de Carvalho Endereço: Universidade Federal da Paraíba/CCS/Departamento de Fisioterapia

Campus Universitário I, s/n - Cidade Universitária

E-mail: sandracordeiror@yahoo.com.br 